\title{
Planificación de la infraestructura - señalización de paradas en la ciudad de Riobamba, aplicando modelos matemáticos.
} Planning of the Infrastructure - signage of stops in the city of Riobamba,
applying mathematical models.

Jenny Margoth Villamarín Padilla. ${ }^{1}$, Monserrath Amparo Padilla Muñoz. ${ }^{2}$, Alexandra Patricia Guerrero Godoy. ${ }^{3}$ \& Guido Javier Mazón Fierro. ${ }^{4}$

Recibido: 10-03-2019 / Revisado: 15-03-209 /Aceptado: 04-04-2019/ Publicado: 13-05-2019

\section{Abstract. \\ DOI: https://doi.org/10.33262/cienciadigital.v3i2.2.459}

The effective displacement by its different road arteries, of the city of Riobamba has many drawbacks because of not having an updated planning of the route of the public transport units, the problem is both for the drivers and pedestrians of the City, for which we allow ourselves to carry out an analysis of the current situation of the infrastructure of the stops and signaling of the different lines that work daily. The GAD (autonomous Municipal Government of the city of Riobamba) as a transit manager in the city of Riobamba must take corrective actions in the road infrastructure, with the execution of projects linked to this drawback to short and medium Term. Taking actions of improvements to a short term according to the magnitude of the problem encountered, another of the problems encountered by the lack of corrective in road planning is its influence on the quality of service urban transportation of the city, as transport is considered the economic engine whose results are reflected in the positive impacts both in the local economy of each city.

1 Escuela Superior Politécnica del Chimborazo, Facultad de Administración de Empresas, Riobamba Ecuador, nyvillamarin@yahoo.es

2 Escuela Superior Politécnica del Chimborazo, Facultad de Administración de Empresas, Riobamba Ecuador, monspath.23@gmail.com

3 Escuela Superior Politécnica del Chimborazo, Facultad de Administración de Empresas, Riobamba Ecuador, patyzzu@ @otmail.com

4 Escuela Superior Politécnica del Chimborazo, Facultad de Administración de Empresas, Riobamba Ecuador, guido.mazon@espoch.edu.ec 
Key words: Urban transport. Quality, service, infrastructure, stops, signage, mathematical models

\section{Resumen}

El desplazamiento efectivo por sus diferentes arterias viales, de la ciudad de Riobamba tiene muchos inconvenientes por no tener una planificación actualizada del recorrido de las unidades de transporte público, el problema se tanto para los conductores y peatones de la ciudad, para el cual nos permitimos realizar un análisis de la actual situación de la infraestructura de las paradas y señalización de las diferentes líneas que laboran diariamente. El GAD (Gobierno Autónomo Descentralizado Municipal de la ciudad de Riobamba) como gestor de tránsito en la ciudad de Riobamba deben tomar acciones correctivas en la infraestructura vial, con la ejecución de proyectos vinculados a este inconveniente a corto y mediano plazo. Tomando las acciones de mejoras a un corto plazo de acuerdo a la magnitud de la problemática encontrada, otro de los problemas encontrados por la falta de correctivos en la planificación vial es su influencia en la calidad del servicio transporte urbano de la ciudad, pues el transporte es considerado el motor económico cuyos resultados se refleja en los impactos positivos tanto en la economía local de cada ciudad.

Palabras clave: Transporte Urbano. Calidad, Servicio, infraestructura, Paradas, señalización, Modelos Matemáticos

\section{Introducción}

La ciudad de Riobamba, ubicada en la zona central de la Región Interandina del Ecuador, pese a esta ubicada en una superficie plana, los inconvenientes para el desplazamiento efectivo por sus diferentes arterias viales, producto de una incorrecta planificación vial, lo que genera inconvenientes a conductores y peatones que demandan de las autoridades mejoras en cuanto tiene que ver con el desplazamiento de las unidades de transporte público, por lo que se hace necesario efectuar un análisis de la actual situación de la infraestructura con que cuenta para el desplazamiento por los diferentes corredores viales. El mal estado de la capa de rodadura en las arterias viales de la ciudad, siendo más notorio este desperfecto en aquellas vías por las cuales circula el trasporte público, factor este que incide sobre manera en la calidad del servicio, el descuido en cuanto tiene que ver con el 
mantenimiento vial y el excesivo tránsito provoca que los tiempos de viaje sean más extensos y peligrosos, lo que genera a la vez insatisfacción en los usuarios por la afectación a su economía, ya sea porque deben emplear el servicio de taxis para llegar oportunamente a sus destinos o por la afectación que la congestión provoca en diferentes ámbitos del convivir diario de la población.

Los baches de las vías originan un deterioro rápido de las unidades de transporte público, suscitando inconvenientes con las cooperativas y compañías que brindan este servicio en la ciudad, lo cual conllevaría a una mala calidad en el servicio de transporte público.

Al ser el Gobierno Autónomo Descentralizado Municipal de la ciudad de Riobamba, el ente gestor del tránsito en la ciudad al haber asumido por ley las competencias en este ámbito, debe emprender acciones que permitan corregir las falencias presentes en la infraestructura vial de la ciudad, partiendo de la evaluación y mantenimiento rutinario y periódico y la ejecución de proyectos vinculados a esta deficiencia, que a corto y mediano plazo permitirán elevar la vida útil de la calzada, garantizar la fluidez del tráfico y mejorar el grado de satisfacción ciudadana con la gestión institucional en este campo.

Ante esta situación, es imprescindible que la Dirección de Gestión de Movilidad, Tránsito y Transporte del GAD Municipal de Riobamba, en conjunto con la Dirección de Gestión de Planificación y Proyectos de la misma entidad prioricen la señalización tanto horizontal como vertical en las vías, toda vez que la deficiente e insuficiente señalización, dificulta la identificación de las paradas del transporte público y limita la calidad de este servicio toda vez que es común el irrespeto de las mismas tanto por parte de los usuarios como de los conductores, cuyo resultado final suele ser diversos accidentes de tránsito, que generan a su múltiples afectaciones económicas, sociales, psicológicas y físicas a los involucrados.

Los problemas que observamos en la infraestructura vial del transporte ocurren a nivel mundial, ningún país se ha estado exento de tratar estos inconvenientes, aunque su dimensión y contexto sean diferentes, las búsquedas de soluciones para satisfacer necesidades son similares.

A continuación, se citan algunas soluciones que han dado diferentes autores a esta problemática: 
La nueva política de la UE, se centra estrictamente en la financiación del transporte en la red principal de transportes, cubriendo las conexiones transfronterizas que faltan, eliminando cuellos de botella y haciendo que la red sea más inteligente. (Comisión Europea, 2011)

La nueva red principal RTE-T se apoya en una red global de líneas nacionales y regionales unida a la red principal. La financiación correrá en gran parte a cargo de los Estados miembros, con algunas posibilidades de financiación regional y del transporte de la UE. El objetivo es garantizar que, de forma progresiva y para 2050, la inmensa mayoría de las empresas y los ciudadanos europeos no necesite más de treinta de minutos de viaje para acceder a la red capilar. (Comisión Europea, 2011)

En nuestro país, la Provincia de Morona Santiago da como solución la reconstrucción y mantenimiento de la vía Méndez - San José de Morona, provincia de Morona Santiago.

\section{Análisis de las vías de la ciudad de Riobamba}

Según la información del VII Censo de Población y VI de Vivienda del año 2010, la provincia de Chimborazo cuenta con una población de 458.581 habitantes, de los cuales 187.119 habitantes viven en el área urbana (40.80\%) y 271462 habitantes viven en el área rural (59.20\%). Para efecto de análisis de acuerdo a la proyección de la población, se estima que la infraestructura vial cuenta con una vida útil de 20 años. (Subsecretaría regional 3, 2014)

Puesto que, en la rehabilitación de las vías de circulación de la ciudad de Riobamba, se ha realizado obras de infraestructura vial en un:

- $100 \%$, constituido por capa de rodadura con pavimento rígido.

- Parterre central,

- Aceras y bordillos,

- Iluminación y semaforización;

En el componente ambiental se cuenta con arborización en un 100\%; El paso vehicular elevado en las Av. Lizarzaburu y la Av. La Prensa el mismo que cuenta con la infraestructura (Cimentación, Queisson y estribos) y en ejecución obras de superestructura (Encofrado, colocación de hierro para las vigas, etc.), señalización del 
puente, iluminación y accesos al puente. Drenaje: Implementado 100\%. Pasos peatonales elevados.

\section{Objetivos}

\section{Objetivo general}

Evaluar la infraestructura vial de los corredores del transporte público de la ciudad de Riobamba y la calidad del servicio.

\section{Objetivos específicos}

- Levantar información de campo de la infraestructura vial referente a señalización y paradas de los corredores de las rutas del transporte público de la ciudad de Riobamba.

- Realizar un diagnóstico de la infraestructura vial de los corredores de las rutas del transporte público de la ciudad de Riobamba.

- Proponer alternativas de mejora de la infraestructura vial de los corredores de las rutas del transporte público de la ciudad de Riobamba.

\section{Materiales y métodos.}

\section{Modalidad de la Investigación}

\section{Cuantitativa}

En la investigación se da el diseño ya que se tomará información que se puede analizar, contabilizar y consecuentemente tener una base de datos para analizar el mismo que se utilizará para los fines de la investigación (Rodriguez, y otros, 2009).

Podemos encontrar la siguiente investigación:

\section{No Experimental}

Dentro de la modalidad de investigación se va a utilizar el diseño no experimental porque solo se emplea observación y que realizara sin manipular deliberadamente variables, es decir, observar fenómenos tal y como se dan en su contexto natural, para después analizarlos y que el investigador no provocara ninguna situación del lugar de estudio (Rodriguez, y otros, 2009).

\section{Cualitativo}


Algunas características serán tomadas del lugar de investigación y los esquemas que se realizan dentro del lugar de investigación por lo que el investigador realizara la toma la información (Rodriguez, y otros, 2009).

\section{Tipos de Investigación}

\section{Investigación Exploratoria}

El contacto mismo del problema para poder estudiarlo, es decir, con esto obtenemos información de carácter seguro para poder seguir con una investigación a fondo.

\section{Investigación Descriptiva}

Utilizaremos de este tipo para poder interpretar, analizar los resultados obtenidos con los instrumentos que utilicemos en la investigación, y plasmarlos para el entendimiento de todos los aspectos que se trate.

\section{Investigación Explicativa}

Busca el inicio del problema y las integra, dando, conclusiones, explicaciones e incrementar del conocimiento del presente trabajo.

\section{Investigación de campo}

Se lleva a cabo en el estudio Campo o estudio natural que se realiza el estudio, es decir, porque el investigador va a observar reacciones ante las diferentes situaciones reales.

\section{Investigación bibliográfica}

Basa su investigación en documentos, repositorios, páginas Web para poder ampliar las bases de la investigación y lo hará referencia a su respectiva norma (APA, 2016).

\section{Investigación Documental}

La recopilación de información en diversas fuentes de revistas, videos para poder recuperar, analizar, criticar e interpretar los datos obtenidos y registrados por anteriores investigadores que han realizado la investigación respecto a las variables a considerar en esta investigación.

\section{Fundamentación teórica.}

\section{Administración}

Para (Ortiz, 2009). La administración es "Proceso integral para planear, organizar e integrara una actividad o relación de trabajo, la que se fundamenta en la utilización de recursos para alcanzar un fin determinado". 
La administración también puede definirse como la planificación, la dirección, la organización y el control de las actividades dentro de una organización para la cual se requerirá, talento humano, recursos económicos, tecnológicos para alcanzar las metas y poder mantenerse en el mercado competitivo.

La administración se aplica para todos los niveles de organización como compañías, cooperativas tales como públicas y privadas con el fin de sacar un mejor rendimiento en términos de eficiencia y eficacia.

\section{Principios generales de la administración}

Cumplir los principios es una forma más eficiente se necesita que facilite el control, poder realizarlos de una forma inteligente, con experiencia, decisión y mesura. Un principio el cual se requiere es la Coordinación, el cual explica, que se refiere a la forma en que se realiza las acciones en la empresa, como pueden ser los problemas en este caso dar las soluciones y no aumentarlos.

\section{De tráfico}

La ingeniería de transporte deriva de la ingeniería civil y es una profesión interdisciplinaria requerida en cualquier tipo de organización, pública o privada, donde es necesario implementar y administrar sistemas de transporte a nivel nacional e internacional proporcionando soluciones a la problemática que se presenta en los diferentes modos: aéreo, terrestre, ferroviario y marítimo. (Universia, 2013)

Ingeniería de tránsito se define como la rama de la ingeniería que trata del planeamiento de las calles, carreteras, zonas anexas a ellas, del proyecto de sus características geométricas y de la circulación del tránsito en las mismas en vistas a su empleo para transportar personas en forma segura económica y cómoda. (Peña, Herlisseth, 2016)

\section{Seguridad vial}

Es el conjunto de mecanismos y acciones que ayuda a garantizar el correcto funcionamiento de la circulación del tránsito, a través de la utilización de conocimientos (reglamento, leyes y disposiciones) y normas de conducta, ya sean estas actuadas como pasajero, peatón o conductor, con el único fin de utilizar de buena forma la vía pública logrando así prevenir siniestros de tránsito. (e-mediadrive, s.f) 


\section{Ciudades}

Ha sido frecuente aproximarse a la dinámica de las ciudades por medio del examen de la evolución de la estructura urbana según el tamaño de las localidades. En esta oportunidad, el tratamiento de la trayectoria sociodemográfica de los sistemas de ciudades reconocerá tres categorías de asentamientos urbanos:

\section{Desarrollo de la investigación}

Tabla1. Inventario de infraestructura de paradas y señalización faltantes líneas $1,2,3$.

\begin{tabular}{|c|c|}
\hline I'TEM & DETALLE \\
\hline 1 & SANTA ANITA \\
\hline 2 & UNIANDES-HORNOS ANDINO \\
\hline 3 & PATIOS ROSALES-REDONDEL CANONIGO \\
\hline 4 & ANDAQUIRRE \\
\hline 5 & PARQUE BARRIGA \\
\hline 6 & TERMINAL LLANTAS CONTIENTAL \\
\hline 7 & EX REDONDEL BYPASS \\
\hline 8 & HOSPITAL ANDINO \\
\hline 9 & BY PASS \\
\hline 10 & PATIO ROSALES \\
\hline 11 & URUGUAY-CARABOBO \\
\hline 12 & LOJA -OROZCO \\
\hline 13 & OROZCO-MEXICO \\
\hline 14 & CISNROS POLICIA \\
\hline 15 & OROZCO ESPEJO \\
\hline 16 & GARCIA MORENO \\
\hline 17 & TERMINAL ANDAGUIRRE \\
\hline 18 & PATIO ROSALES-HORNOS ANDINOS \\
\hline 19 & SEMAFOROS ACACIAS \\
\hline 20 & ECU 911 \\
\hline 21 & TANQUES DE AGUA \\
\hline
\end{tabular}




$\begin{array}{ll}22 & \text { REDONDEL BCO. GUYAQUIL } \\ 23 & \text { ESTADIO-ESCALADA } \\ 24 & \text { LOJA-PURUHA } \\ 25 & \text { INGRESO FAUSTO MOLINA } \\ 26 & \text { ECUAVISION } \\ 27 & \text { ESCUELA FE Y -VELASCO } \\ 28 & \text { COLON } \\ 29 & \text { ROCAFUERET } \\ 30 & \text { PLAZZA DE TOROS } \\ 31 & \text { GASOLINERA PARQUE INFANTIL } \\ 32 & \text { TANQUES DE AGUA } \\ 33 & \text { EX REDONDEL BY PASS } \\ & \text { SEMAFOROS ACACIAS }\end{array}$

Fuente: Investigación de campo

Elaboración: Grupo de Investigación

Tabla2. Inventario de infraestructura de paradas y señalización faltantes líneas 4,5,6.

\begin{tabular}{ccc}
\hline ÍTEM & \multicolumn{2}{c}{ DETALLE } \\
\hline 1 & LICAN & ESTADIO LICAN \\
2 & EX MEDIA LUNA & ENTRADA TIERRA NUEVA \\
3 & ENTRADA TIERRA NUEVA & ESPOCH \\
4 & SAINT AMAUND M. & GASOLINERA SINDICATRO \\
5 & GASOLINERA SINDICATRO & VELASCO \\
7 & CRISTOBAL COLON & FAUSTO MOLINA \\
8 & UNACH & COLEGIO DAQUILEMA \\
9 & FAUSTO MOLINA & 5 DE JUNIO \\
10 & JUNIN & COLISEO \\
11 & ESPEJO & ESTADIO LICAN \\
\hline & EX MEDIA LUNA & \\
\hline
\end{tabular}


GAUSHI

EX MEDIA LUNA

U.E JUAN DE VELASCO

MÉXICO

5 DE JUNIO

EX MEDIA LUNA

ESTADIO LICAN

CORONA REAL

CUNDUANA

ENTRADA TIERRA NUEVA

PUERTA ING AGRONOMÍA

PUERTA ING ED PRINCIPAL

SAINT AMAUND M.

POLICÍA

05-jun

OROZCO

ESPOCH

PUERTA ING ED PRINCIPAL

PUERTA ING AGRONOMÍA

ENTRADA TIERRA NUEVA

EX MEDIA LUNA

ESTADIO LICÁN
CUNDUANA

TENTRADA TIERTRA NUEVA

MÉXICO

JUNIN

OROZCO

ESTADIO LICAN

CORONA REAL

CONDUANA

GAUSHI

PUERTA ING AGRONOMÍA

PUERTA ING ED PRINCIPAL

ESPOCH

REDONDEL DEL LIBRO

CISNEROS

OROZCO

ESPEJO

PUERTA ING ED PRINCIPAL

PUERTA ING AGRONOMÍA

ENTRADA TIERRA NUEVA

EX MEDIA LUNA

ESTADIO LICÁN

LICÁN

Fuente: Investigación de campo

Elaboración: Grupo de Investigación

Tabla3 Inventario de infraestructura de paradas y señalización faltantes línea 9

\begin{tabular}{clc}
\hline ITEM & DETALLE \\
\hline 1 & LICAN & ESTADIO DE LICAN \\
2 & MEDIA LUNA & ENTRADA A TIERRA NUEVA \\
3 & ENTRADA A TIERRA NUEVA & ESPOCH \\
\hline
\end{tabular}




\begin{tabular}{|c|c|c|}
\hline 4 & MILTON REYES & ANDAGUIRRE \\
\hline 5 & SEMAFORO COL. RIOBAMBA & CARLOS ZAMBRANO \\
\hline 6 & CARLOS ZAMBRANO & FISCALIA \\
\hline 7 & CARABOBO & ESPAÑA \\
\hline 8 & ESPAÑA & GUAYAQUIL \\
\hline 9 & GUAYAQUIL & COLOMBIA \\
\hline 10 & COLOMBIA & COL. ISABEL DE GODIN \\
\hline 11 & LA PAZ & HOSPITAL \\
\hline 12 & HOSPITAL & REDONDEL VIA SAN LUIS \\
\hline 13 & REDONDEL VIA SAN LUIS & CAMAL \\
\hline 14 & CAMAL & AV. BOLIVAR BONILLA \\
\hline 15 & AV. BOLIVAR BONILLA & ECUAVISION \\
\hline 16 & POLICIA & CISNEROS \\
\hline 17 & LOJA & DIRECCION DE MOVILIDAD \\
\hline 18 & DIRECCION DE MOVILIDAD & ENTRADA CAMILO PONCE \\
\hline 19 & ENTRADA CAMILO PONCE & AV. CORDOVEZ \\
\hline 20 & AV. CORDOVEZ & NEW YORK \\
\hline 21 & NEW YORK & CARABOBO \\
\hline 22 & CARABOBO & URUGUAY \\
\hline 23 & URUGUAY & SALESIANOS \\
\hline 24 & SALESIANOS & ESC. PRIMERA CONSTITUYENTE \\
\hline 25 & ESC. PRIMERA CONSTITUYENTE & TERMINAL \\
\hline 26 & TERMINAL & ANDAGUIRRE \\
\hline 27 & ANDAGUIRRE & MILTON REYES \\
\hline 28 & MILTON REYES & ESPOCH \\
\hline 29 & ENTRADA A TIERRA NUEVA & EX MEDIA LUNA \\
\hline 30 & EX MEDIA LUNA & ESTADIO DE TICAN \\
\hline 31 & ESTADIO DE LICAN & LICAN \\
\hline
\end{tabular}

Fuente: Investigación de campo

Elaboración: Grupo de Investigación 
Tabla 4. Inventario de infraestructura de paradas y señalización faltantes línea 10

\begin{tabular}{|c|c|c|}
\hline ÍTEM & \multicolumn{2}{|c|}{ DETALLE } \\
\hline 1 & LANGOS SAN MIGUEL & SAN ANTONIO \\
\hline 2 & SAN ANTONIO & UNACH \\
\hline 3 & UNACH & COLEGIO MALDONADO \\
\hline 4 & COLEGIO MALDONADO & COMIL \\
\hline 5 & COMIL & EX QUITO MOTORS \\
\hline 6 & EX QUITO MOTORS & LOMA DE QUITO \\
\hline 7 & LOMA DE QUITO & SAN ALFONSO \\
\hline 8 & SAN ALFONSO & SAN FRANCISCO \\
\hline 9 & SAN FRANCISCO & DOLOROSA \\
\hline 10 & DOLOROSA & FAUSTO MOLINA \\
\hline 11 & FAUSTO MOLINA & ANT \\
\hline 12 & LOJA & COLEGIO ISABEL DE GODIN \\
\hline 13 & COLEGIO ISABEL DE GODIN & VILLARROEL \\
\hline 14 & VILLARROEL & CARABOBO \\
\hline 15 & CARABOBO & CONDAMINE \\
\hline 16 & CONDAMINE & FRANCIA \\
\hline 17 & FRANCIA & COLOMBIA \\
\hline 18 & COLOMBIA & NICANOR LARREA \\
\hline 19 & TERMINAL & REDONDEL DEL AEROPUERTO \\
\hline 20 & REDONDEL DEL AEROPUERTO & SALESIANOS \\
\hline 21 & SALESIANOS & EX QUITO MOTORS \\
\hline 22 & EX QUITO MOTORS & SEMAFOROS HEROES DE TAPI \\
\hline 23 & SEMAFOROS HEROES DE TAPI & COMIL \\
\hline 24 & COMIL & COLEGIO MALDONADO \\
\hline 25 & COLEGIO MALDONADO & UNACH \\
\hline 26 & SAN ANTONIO & LANGOS SAN MIGUEL \\
\hline
\end{tabular}

Fuente: Investigación de campo

Elaboración: Grupo de Investigación 


\section{Acciones de Mejoras}

Tabla 5. Acciones de Mejoras

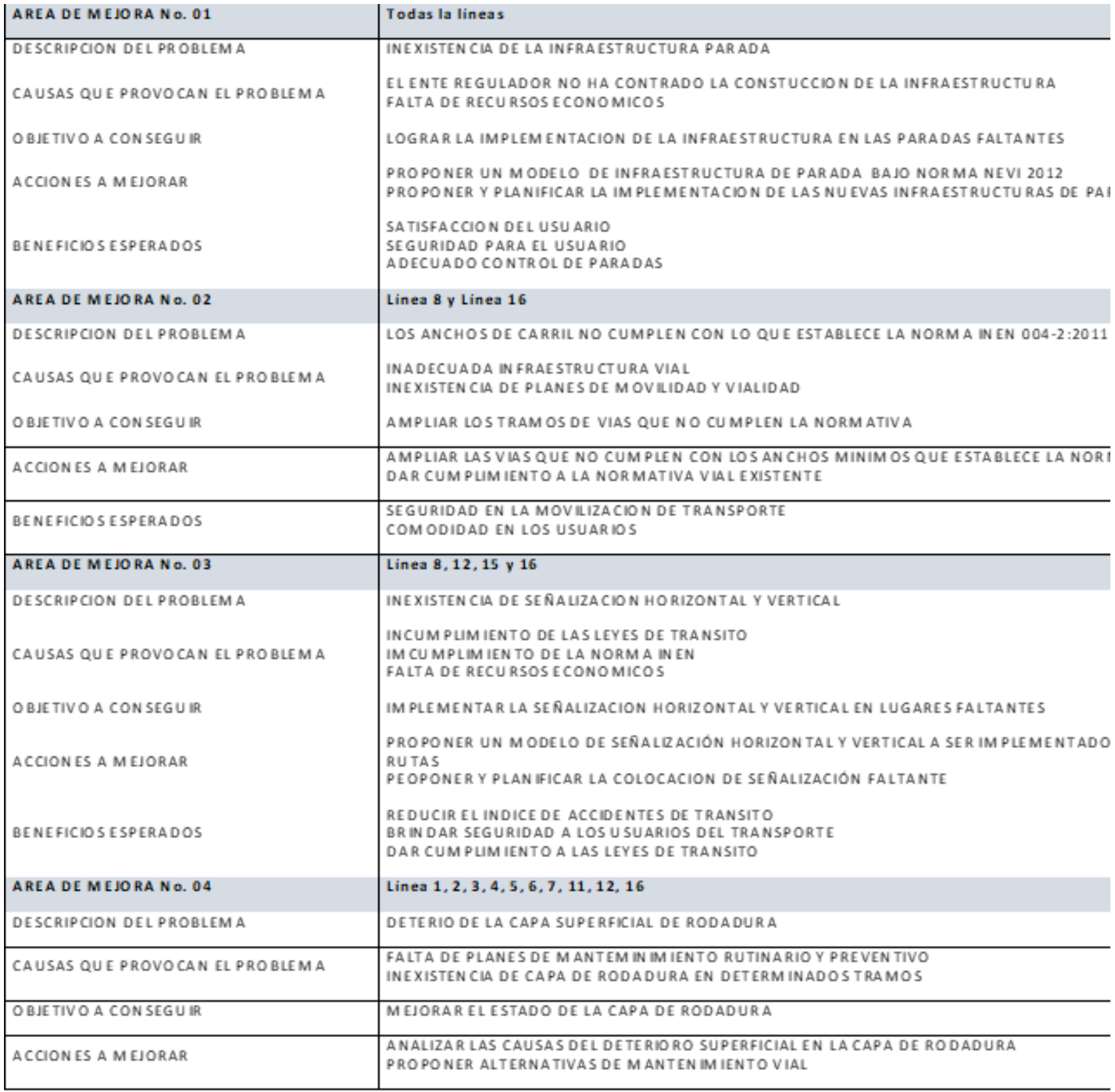

Fuente: Investigación de campo

Elaboración: Grupo de Investigación

Análisis: Todas las líneas muestran problemas en diferentes áreas las misma que deben ser analizados inmediatamente por el ente rector.

Análisis de la Calidad del Servicio. 


\section{Resultados y Discusión.}

Datos arrojados del plan de mejoras por áreas

AREA DE MEJORA No. 01 Todas las líneas

DESCRIPCION DEL PROBLEMA inexistencia de la infraestructura parada

CAUSAS QUE PROVOCAN EL PROBLEMA el ente regulador no ha contratado la construcción de la infraestructura

FALTA DE RECURSOS ECONOMICOS

OBJETIVO A CONSEGUIR, lograr la implementación de la infraestructura en las paradas faltantes

ACCIONES A MEJORAR proponer un modelo de infraestructura de parada bajo norma nevi 2012.

AREA DE MEJORA No. 02 Línea 8 y Línea 16

DESCRIPCION DEL PROBLEMA los anchos de carril no cumplen con lo que establece la norma inen 004-2:2011 y mtop

CAUSAS QUE PROVOCAN EL PROBLEMA inadecuada infraestructura vial. inexistencia de planes de movilidad y vialidad OBJETIVO A CONSEGUIR AMPLIAR LOS TRAMOS DE VIAS QUE NO CUMPLEN LA NORMATIVA

ACCIONES A MEJORAR ampliar las vías que no cumplen con los anchos mínimos que establece la norma dar cumplimiento a la normativa vial existente beneficios esperados seguridad en la movilización de transporte comodidad en los usuarios proponer y planificar la implementación de las nuevas infraestructuras de parada beneficios esperados satisfacción del usuario seguridad para el usuario adecuado control de paradas.

AREA DE MEJORA No. 03 Línea 8, 12, 15 y 16

DESCRIPCION DEL PROBLEMA inexistencia de señalización horizontal y vertical CAUSAS QUE PROVOCAN EL PROBLEMA incumplimiento de las leyes de transito

IMCUMPLIMIENTO DE LA NORMA INEN

FALTA DE RECURSOS ECONOMICOS 
OBJETIVO A CONSEGUIR implementar la señalización horizontal y vertical en lugares faltantes

ACCIONES A MEJORAR proponer un modelo de señalización horizontal y vertical a ser implementado en las rutas proponer y planificar la colocación de señalización faltante beneficios esperados reducir el índice de accidentes de tránsito brindar seguridad a los usuarios del transporte dar cumplimiento a las leyes de transito

AREA DE MEJORA No. 04 Línea 1, 2, 3, 4, 5, 6, 7, 11, 12, 16

DESCRIPCION DEL PROBLEMA deterior de la capa superficial de rodadura causas que provocan el problema falta de planes de mantenimiento rutinario y preventivo inexistencia de capa de rodadura en determinados tramos

OBJETIVO A CONSEGUIR mejorar el estado de la capa de rodadura

acciones a mejorar analizar las causas del deterioro superficial en la capa de rodadura proponer alternativas de mantenimiento vial beneficios esperados comodidad en la circulación

reducción de accidentes de tránsito satisfacción del usuario reducción de costos directos del transportista

\section{Conclusiones.}

El análisis de infraestructura en todas las líneas nos da como resultados la falta de señalética informativa, inexistencia de la infraestructura parada en las líneas 8 y 16 los anchos de carril no cumplen con lo que establece la norma inen 004-2:2011 y mtop se propone planificar la implementación de las nuevas infraestructuras de parada beneficios esperados satisfacción del usuario seguridad para el usuario adecuado control de paradas. Línea 8, 12, 15 y 16 se encontró inexistencia de señalización horizontal y vertical se propone un modelo de señalización horizontal y vertical a ser implementado en las rutas proponer y planificar la colocación de señalización faltante,

Línea 1, 2, 3, 4, 5, 6, 7, 11, 12, 16 se encontró deterior de la capa superficial de rodadura se propone proponer alternativas de mantenimiento vial beneficios esperados comodidad en la circulación reducción de accidentes de tránsito satisfacción del usuario reducción de costos directos del transportista. 
En cuanto al análisis de calidad podemos concluir que no es un servicio de calidad pues en tiempo de espera en las líneas que no son céntricas es decir líneas alejadas el tiempo de espera demora más de 7 minutos y pasan a más de 5 cuadras en algunos casos y no existen paradas definidas peor aún señalética informativa para el usuario.

\section{Análisis.}

Todas las líneas muestran problemas en diferentes áreas las misma que deben ser analizados inmediatamente por el ente rector. El deterior de la capa superficial de rodadura se propone proponer alternativas de mantenimiento vial beneficios esperados comodidad en la circulación reducción de accidentes de tránsito satisfacción del usuario reducción de costos directos del transportista.

En cuanto al análisis de calidad podemos concluir que no es un servicio de calidad pues en tiempo de espera en las líneas que no son céntricas es decir líneas alejadas el tiempo de espera demora más de 7 minutos y pasan a más de 5 cuadras en algunos casos y no existen paradas definidas peor aún señalética informativa para el usuario.

\section{Bibliografía}

Cronin, J., \& Taylor, S. (1992). Measuring Service Quality: A Reexamination and Extension. American Marketing Association, 55-68.

e-mediadrive. (s.f). ¿Qué es seguridad vial? Obtenido de http://culturavial.com/seguridadvial/que-es-seguridad-vial.html

GOMEZ, R. C. (2015). ADMINISTRACION DE LA CALIDAD TOTAL. MAR DE PLATA: UNIVERSIDAD MAR DE PLATA.

Peña, Herlisseth. (2016). Ingenieria de transito. Obtenido de https://es.slideshare.net/herlissethsaia/ingenieria-de-transito-59141921

Rodriguez, D., \& Valldeoriola, J. (2009). Metodologia de Investigación. Barcelona: Universitat Oberta de Catalunya.

Salas r, a. G. (2018). ANÁLISIS MATEMÁTICO EN LA CALIDAD EN EL TRANSPORTE URBANO EN LA CIUDAD DE RIOBAMBA PARA FUTUROS PROYECTOS DE INVERSIÓN PÚBLICA. https://www.eumed.net/rev/caribe/index.html, 23.

Universia. (2013). ¿Qué hacen los ingenieros de transporte? Obtenido de http://noticias.universia.net.mx/empleo/noticia/2013/05/20/1024544/que-haceningenieros-transporte.html 
Valarie, Parasuraman, \& Berry. (1993). Calidad total en la gestión de servicios. Madrid: Diaz de Santos. 


\section{PARA CITAR EL ARTÍCULO INDEXADO.}

Villamarín Padilla, J., Padilla Muñoz, M., Guerrero Godoy, A., \& Mazón Fierro, G. (2019). Planificación de la infraestructura - señalización de paradas en la ciudad de Riobamba, aplicando modelos matemáticos. Ciencia Digital, 3(2.2), 48-65.

https://doi.org/10.33262/cienciadigital.v3i2.2.459

\section{Ciencia
Digital
Editabal}

El artículo que se publica es de exclusiva responsabilidad de los autores y no necesariamente reflejan el pensamiento de la Revista Ciencia Digital.

El artículo queda en propiedad de la revista y, por tanto, su publicación parcial y/o total en otro medio tiene que ser autorizado por el director de la Revista Ciencia Digital.
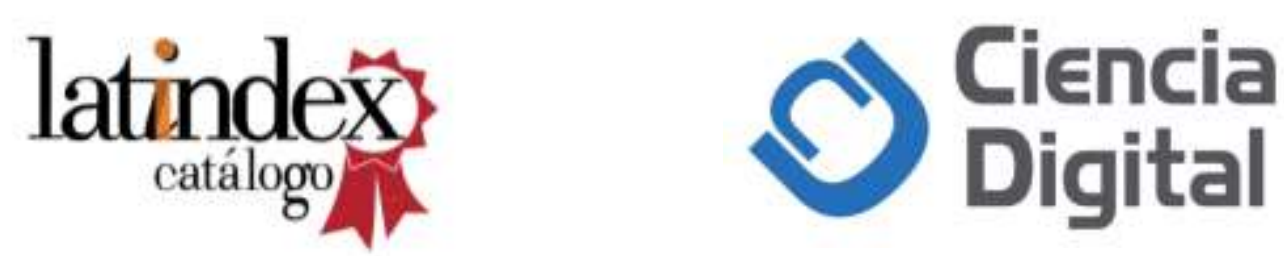